\title{
Erratum to: Is body mass index a good indicator of obesity?
}

\author{
Shriraam Mahadevan ${ }^{1} \cdot$ Iftikhar $^{\mathrm{Ali}^{2,3}}$
}

Published online: 14 June 2016

(C) Research Society for Study of Diabetes in India 2016

Erratum to: Int J Diabetes Dev Ctries

DOI 10.1007/s13410-016-0506-5

Dr. Iftikhar Ali was missing in the original version of this article and should be included as co-author.

The online version of the original article can be found at http://dx.doi. org/10.1007/s13410-016-0506-5.

$\triangle$ Shriraam Mahadevan mshriraam@gmail.com

1 Department of Endocrinology, Diabetes and Metabolism, Sri Ramachandra Medical College, Chennai, India

2 Department of Pharmacy, Northwest General Hospital \& Research Center, Peshawar, Pakistan

3 Department of Pharmacy, University of Swabi, Khyber Pakhtunkhwa, Pakistan 